\title{
Erratum to: Incomplete Sentinel Node Biopsy Is Not Clearly Related to Survival or Regional Recurrence in Cutaneous Melanoma Patients
}

Nicholas C. Lee, MBBS ${ }^{1}$, Andrew J. Spillane, MD ${ }^{1,2,3,4,5}$, Tony C. Y. Pang, MBBS, MBiostat ${ }^{1}$, Lauren E. Haydu, BSCHE, MIPH ${ }^{2,3}$, and Roger F. Uren, PhD $^{2,6}$

${ }^{1}$ Royal North Shore Hospital, St. Leonards, Australia; ${ }^{2}$ Sydney Medical School, The University of Sydney, Sydney, Australia; ${ }^{3}$ Melanoma Institute Australia, Sydney, Australia; ${ }^{4}$ The Mater Hospital, North Sydney, Australia; ${ }^{5}$ Royal Prince Alfred Hospital, Camperdown, Australia; ${ }^{6}$ Nuclear Medicine and Diagnostic Ultrasound, RPAH Medical Centre, Camperdown, Australia

\section{ERRATUM TO: ANN SURG ONCOL}

\section{DOI 10.1245/S10434-011-1872-3}

In the original publication of this article, Table 3 included errors in the SNB Completeness Factor section. The table is reprint here in its entirety correctly.

The online version of the original article can be found under doi:10.1245/s10434-011-1872-3.

(C) Society of Surgical Oncology 2011

A. J. Spillane, MD

e-mail: andrew.spillane@melanoma.org.au 
TABLE 3 Results of univariate survival analysis of categorical variables

\begin{tabular}{|c|c|c|c|c|c|c|c|}
\hline \multirow[t]{2}{*}{ Factor } & \multirow[t]{2}{*}{ Values } & \multicolumn{2}{|c|}{ Melanoma specific } & \multicolumn{2}{|c|}{ Disease-free } & \multicolumn{2}{|c|}{ Regional recurrence-free } \\
\hline & & $N$ & 5-year survival & $N$ & 5-year survival & $N$ & 5-year survival \\
\hline \multirow[t]{3}{*}{ Patient sex } & $P$-value & & $<0.001$ & & 0.051 & & 0.009 \\
\hline & Female & 780 & $92.7 \%$ & 781 & - & 781 & $94.5 \%$ \\
\hline & Male & 1119 & $84.2 \%$ & 1123 & - & 1123 & $90.3 \%$ \\
\hline \multirow[t]{5}{*}{ Thickness } & $P$-value & & $<0.001$ & & $<0.001$ & & $<0.001$ \\
\hline & $0-1 \mathrm{~mm}$ & 260 & $97.7 \%$ & 260 & $95.4 \%$ & 260 & $98.2 \%$ \\
\hline & $1.01-2 \mathrm{~mm}$ & 854 & $93.2 \%$ & 858 & $85.5 \%$ & 858 & $94.3 \%$ \\
\hline & $2.01-4 \mathrm{~mm}$ & 542 & $81.8 \%$ & 543 & $72.0 \%$ & 543 & $89.6 \%$ \\
\hline & $>4 \mathrm{~mm}$ & 223 & $71.8 \%$ & 223 & $63.2 \%$ & 223 & $83.2 \%$ \\
\hline \multirow[t]{3}{*}{ Ulceration } & $P$-value & & $<0.001$ & & $<0.001$ & & $<0.001$ \\
\hline & Absent & 1462 & $91.0 \%$ & 1466 & $83.7 \%$ & 1466 & $93.7 \%$ \\
\hline & Present & 415 & $76.2 \%$ & 416 & $67.2 \%$ & 416 & $86.4 \%$ \\
\hline \multirow[t]{3}{*}{ Mitoses } & $P$-value & & 0.038 & & $<0.001$ & & 0.036 \\
\hline & Absent & 335 & $94.4 \%$ & 335 & $90.2 \%$ & 335 & $96.2 \%$ \\
\hline & Present & 1542 & $86.4 \%$ & 1547 & $78.1 \%$ & 1547 & $91.3 \%$ \\
\hline \multirow[t]{5}{*}{ Sentinel node field } & $P$-value & & 0.053 & & 0.009 & & 0.039 \\
\hline & Axilla (blue) & 836 & - & 838 & $83.2 \%$ & 838 & $93.7 \%$ \\
\hline & Groin (green) & 538 & - & 539 & $78.1 \%$ & 539 & $89.5 \%$ \\
\hline & Neck (tan) & 324 & - & 325 & $75.1 \%$ & 325 & $91.9 \%$ \\
\hline & Other (purple) & 201 & - & 202 & $80.0 \%$ & 202 & $93.0 \%$ \\
\hline \multirow[t]{3}{*}{ SNB completeness } & $P$-value & & 0.056 & & 0.007 & & 0.144 \\
\hline & Complete & 1517 & - & 1521 & $81.2 \%$ & 1521 & - \\
\hline & Incomplete & 382 & - & 383 & $75.1 \%$ & 383 & - \\
\hline
\end{tabular}

